Review Article

\title{
Zebrafish: a novel model in neuropsychopharmacological research
}

\author{
Prasan Ramchandra Bhandari*
}

Department of Pharmacology, S.D.M. College of Medical Sciences and Hospital, Sattur, Dharwad-580009, Karnataka, India

Received: 14 April 2016 Accepted: 07 May 2016

\section{*Correspondence to: \\ Dr. Prasan Ramchandra Bhandari, \\ Email: prasangeeta2012 \\ @ gmail.com}

Copyright: () the author(s), publisher and licensee Medip Academy. This is an openaccess article distributed under the terms of the Creative Commons Attribution NonCommercial License, which permits unrestricted noncommercial use, distribution, and reproduction in any medium, provided the original work is properly cited.

\begin{abstract}
The zebrafish (danio rerio) has recently become a powerful animal model for research purposes and drug discovery due to its ease of maintenance, genetic manipulability and ability for high-throughput screening. It has emerged as a model species for translational research in various neuroscience areas, including pharmacogenetics and neuropharmacology. Due to their physiological (neuroanatomical, neuroendocrine, neurochemical) and genetic homology to mammals, robust phenotypes, and value in high-throughput genetic and chemical genetic screens, zebrafish are ideal for developing valid experimental models of major neuropsychiatric disorders and discovering novel therapeutics. Both larval and adult zebrafish are presently used to enhance our understanding of brain function, dysfunction, and their genetic and pharmacological modulation. This article provides a review of the developing utility of zebrafish in the analysis of complex brain disorders (including, e.g., depression, autism, psychoses, drug abuse, and cognitive deficits), also covering zebrafish applications towards the goal of modelling major human neuropsychiatric and drug-induced syndromes.
\end{abstract}

Keywords: Zebrafish, Model, Neuropsychopharmacology

\section{INTRODUCTION}

Psychiatric disorders are a varied set of diseases that influence all features of mental function including social interaction, thinking, feeling, and mood. Though psychiatric disorders put a huge economic burden on society, the drugs offered to treat them are often palliative with inconsistent efficacy and unbearable side-effects. The development of new drugs has been slowed down by a lack of knowledge about the etiology of these diseases. Hence it is imperative to further explore psychiatric disorders using a combination of human molecular genetics, in vitro pharmacological and biochemistry experiments, animal models, and investigation of the nonbiological basis of these diseases, such as environmental effects. ${ }^{1}$ Furthermore, as the population ages, there is an increasing need for efficacious and safe therapies for the treatment of neurological diseases. A restricted number of treatment options are presently available to recover the cognitive dysfunction and research is limited by the need for in vivo models.

Animal models of these disorders are widely used in behavioural neuroscience to explore brain abnormalities, screen drugs and establish behavioural phenotypes of gene-targeted or transgenic animals. Noting a deficit of fresh ideas and especially new paradigms for animal models, there exists a challenge and a pressing need for important directions for further research in this field. Current models in biological psychiatry focus on a handful of model species, and the majority of work relies on data generated in rodents. However, the inclusion of other species and an adoption of comparative viewpoints in behavioural neuroscience could also lead to increases 
in knowledge relevant to biological psychiatry. To achieve this goal, the current focus on mammalian species must be expanded to include other species, including nonmammalian taxa. ${ }^{2}$

This review will look at the utility of the zebrafish in the study of neuropsychiatric diseases and neuropsychopharmacological research.

\section{Rationale for the use of zebrafish as a novel model in neuropsychopharmacological research}

In the above mentioned scenario, a small aquatic vertebrate, the zebrafish (danio rerio) (Figure 1), is rapidly becoming a new accepted model organism in biomedical research.

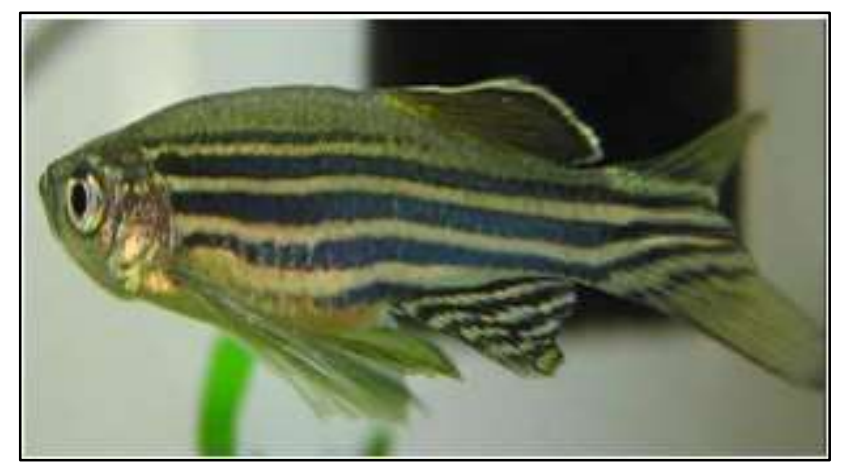

Figure 1: Zebrafish.

Major universities and research centers worldwide have established zebrafish facilities, and the US National Institutes of Health have recently constructed the world's biggest zebrafish center, with the ability to house up to 19 000 tanks and 100000 fish. The usefulness of both adult and larval zebrafish in neuroscience has developed strikingly in the past decades since it is a vertebrate species with high physiological and genetic homology to humans, and also due to the ease of genetic manipulation and comparable central nervous system (CNS) morphology.

Because of its both rapid development (Figure 2) and a relatively long lifespan zebrafish are currently used to model various human brain disorders. Additionally, the close parallels between mammalian and zebrafish behavioral paradigms hint towards the evolutionarily conserved nature of many behaviors (and deficits of their control) across species, suggesting face and construct validity of zebrafish models. Zebrafish are especially cost-efficient, easy to breed, and can be housed in large numbers in a relatively small space. Thus, zebrafish is rapidly becoming an accepted model organism in pharmacogenetics and neuropharmacology. Both larval and adult zebrafish are currently used to increase our understanding of brain function, dysfunction, and their genetic and pharmacological modulation.

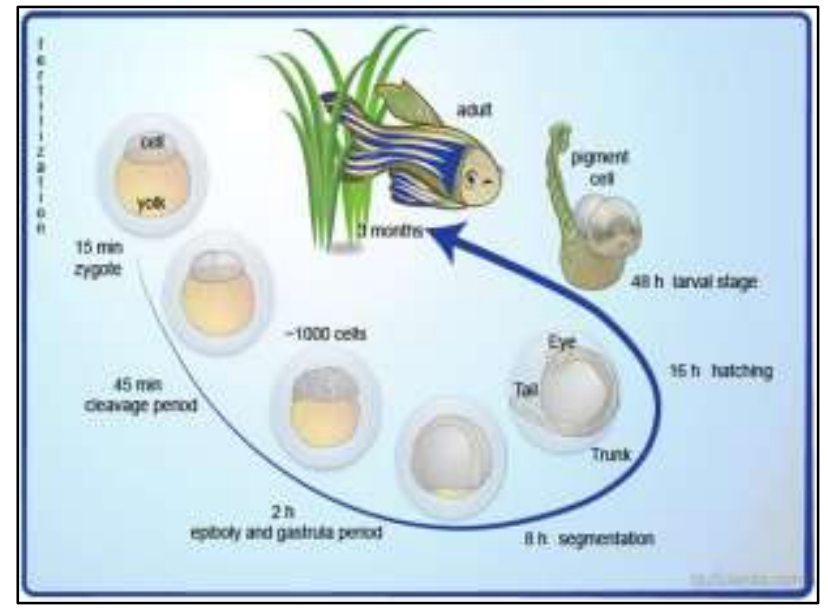

Figure 2: Zebrafish life cycle.

There is a striking similarity between zebrafish and mammalian (rodent) models, including both general macro-organization of the brain and cellular morphology. The knowledge gained from these studies is very relevant to human brain functioning. In addition, zebrafish possess all main neuromediator systems, including transmitters, their receptors, transporters, and enzymes of synthesis and metabolism, similar to those observed in humans and rodents. $^{3}$

Modulatory neurotransmitters which signal through G protein-coupled receptors control brain functions which deteriorate in degenerative brain diseases. In the past decade several of these systems have been mapped in the zebrafish brain. The main structural designs of the systems in zebrafish brain bear a resemblance to that of the mammals, notwithstanding differences in the development of the telencephalon and mesodiencephalon. Modulatory neurotransmitters systems which degenerate in human diseases include dopamine, noradrenaline, serotonin, histamine, acetylcholine and orexin/hypocretin. While the amount of $G$ protein-coupled receptors in zebrafish is evidently larger than in mammals, numerous receptors have similar expression patterns, binding and signaling properties as in mammals. Zebrafish are sensitive to neurotoxins including MPTP (1-methyl-4phenyl-1,2,3,6-tetrahydropyridine), and exposure to this neurotoxin induces a decline in dopamine content and number of detectable tyrosine hydroxylase immune reactive neurons in distinct nuclei. Sensitivity to important neurotoxins, many available genetic methods, rapid development and large-scale quantitative behavioural methods in addition to advanced quantitative anatomical methods render zebrafish an optimal organism for studies on disease mechanisms. ${ }^{4}$

Furthermore, zebrafish are sensitive to all major classes of neurotropic drugs, including antipsychotics, mood stabilizers, anxiolytics, antidepressants, ethanol, sedatives/hypnotics, stimulants, hallucinogens, antiepileptics, anesthetic/analgesics, and cognitive enhancers. ${ }^{3}$ Because of their relatively small size of 
zebrafish larvae, assays can be undertaken in 96 well plates and as the larvae can live in as little as 200 mul of fluid, only a few milligrams of compound are needed for screening. Thus in vivo analysis of the effects of compounds can be undertaken at much earlier stages in the drug discovery process. ${ }^{5}$

It is perceived that zebrafish models of complex brain disorders and drug-induced conditions are a rapidly emerging critical field in translational neuroscience and pharmacology research.

\section{Anxiety and anxiolytics}

Zebrafish is becoming progressively more accepted in neurobehavioral research. By the use of the novel tank test (Figure 3) as a sensitive and efficient behavioural assay, zebrafish anxiety-like behaviour is being capable of bi-directionally altered by drugs affecting the gammaaminobutyric acid, monoaminergic, cholinergic, glutamatergic and opioidergic systems. Complementing human and rodent data, zebrafish drug-evoked phenotypes obtained in this test support this species as a useful model for neurobehavioral and psychopharmacological research. ${ }^{6}$

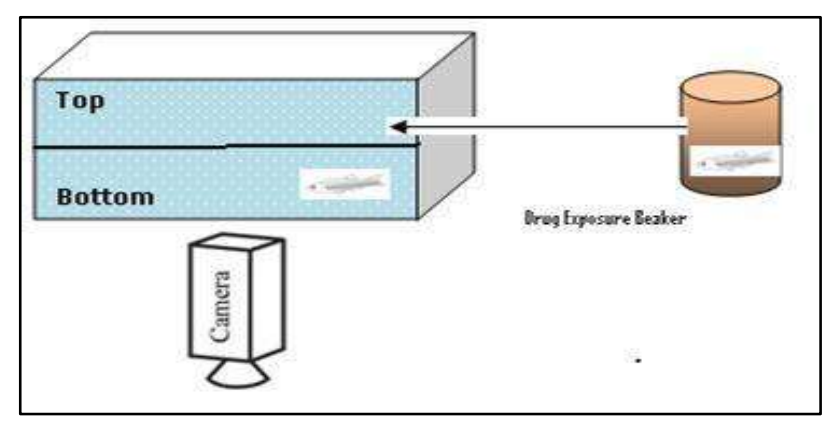

Figure 3: Novel tank test for anxiety.

Cachat $\mathbf{J}$ et al have devised a protocol, and described a battery of assays to characterize anxiety-related behavioural and endocrine phenotypes in adult zebrafish. They have demonstrated how to use the 'novel tank' test to assess behavioural indices of anxiety (including reduced exploration, increased freezing behaviour and erratic movement), which are quantifiable using manual registration and computer-aided video-tracking analyses. Furthermore, authors have described how to analyse whole-body zebrafish cortisol concentrations that correspond to their behaviour in the novel tank test. This protocol is an easy, economical and effective alternative to other methods of quantifying stress responses in zebrafish, thus facilitating the swift acquisition and exploration of large amounts of data. Fish anxiety-like behaviour can be either reduced or exaggerated depending on stress or drug exposure, with cortisol levels generally expected to parallel anxiety behaviour's. This protocol can be accomplished over the course of 2 days, with an adaptable testing duration depending on the number of fish used. ${ }^{7}$
Bencan $\mathrm{Z}$ et al have established a technique to evaluate novel environment diving behaviour of zebrafish as a model of stress response and anxiolytic drug effects. In a novel tank, zebrafish dwell in the bottom of the tank initially and then increase their swimming exploration to higher levels over time. It was earlier established that nicotine, which has anxiolytic effects in rodents and humans, considerably reduces the novel tank diving response in zebrafish. The specificity of the diving effect was corroborated with a novel vs. non-novel test tank. The novel tank diving response of zebrafish was tried when given three anxiolytic drugs from two different chemical and pharmacological classes: buspirone, chlordiazepoxide and diazepam. Buspirone, a serotonergic (5HT(1A) receptor agonist) anxiolytic drug with some $\mathrm{D}(2)$ dopaminergic effect, had a distinct anxiolytic-like effect in the zebrafish diving model at doses that did not have sedative effects. In distinction, chlordiazepoxide, a benzodiazepine anxiolytic drug, which is an effective agonist at GABA-A receptors, did not produce signs of anxiolysis in zebrafish over a broad dose range up to those that caused sedation. Diazepam another benzodiazepine anxiolytic drug did produce an anxiolytic effect at doses that did not cause sedation. The zebrafish novel tank diving task can be useful in discerning anxiolytic drugs of several classes (serotonergic, benzodiazepines and nicotinic). ${ }^{8}$

Current data has established behavioural responses of adult zebrafish to a varied gamut of putative anxiolytic and anxiogenic agents. Experimental manipulations encompassed exposure to alarm pheromone, chronic exposure to fluoxetine, acute exposure to caffeine, as well as acute and chronic exposure to ethanol. Acute (but not chronic) alarm pheromone and acute caffeine produced significant anxiogenic effects, including reduced exploration, augmented erratic movements and freezing behaviour in zebrafish tested in the novel tank diving test. However, ethanol and fluoxetine had robust anxiolytic effects, comprising higher exploration and decreased erratic movements. (Figure 4) Additionally, investigators have established a simple and effective method of assessing zebrafish physiological stress responses (based on a human salivary cortisol assay), and presented that alterations in whole-body cortisol levels in zebrafish parallel behavioural indices of anxiety. ${ }^{9}$

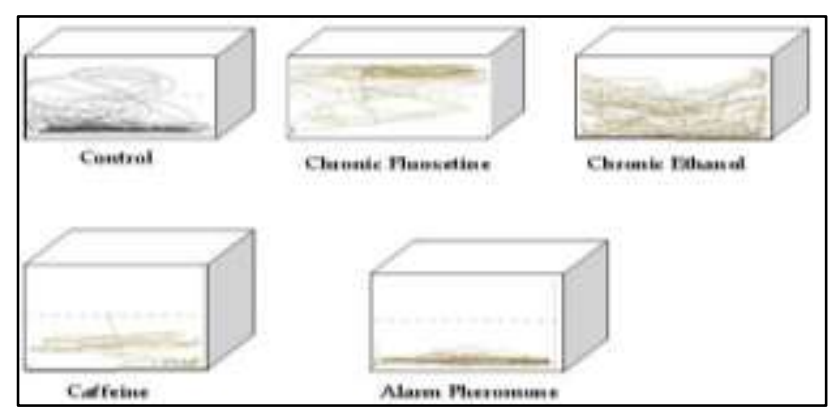

Figure 4: Zebrafish anxiety/anxiolytic responses. 
The chronic unpredictable stress (CUS) paradigm is a commonly used model of experimental stress, wherein rodents are exposed to a series of chronic stressors, like restraint, crowding, isolation, novelty, temperature change, light, noise, and/or predator exposure (Figure 5). Current studies have effectively applied CUS in zebrafish, which affects shoaling, exploration, and anxiety behaviours, besides modifying brain proteome profiles and neurogenesis (the hallmark of affective disorders in rodent models); they also demonstrate chronic stressinduced memory deficits and raised cortisol levels, similar to depression-like states in humans and rodents. ${ }^{10}$

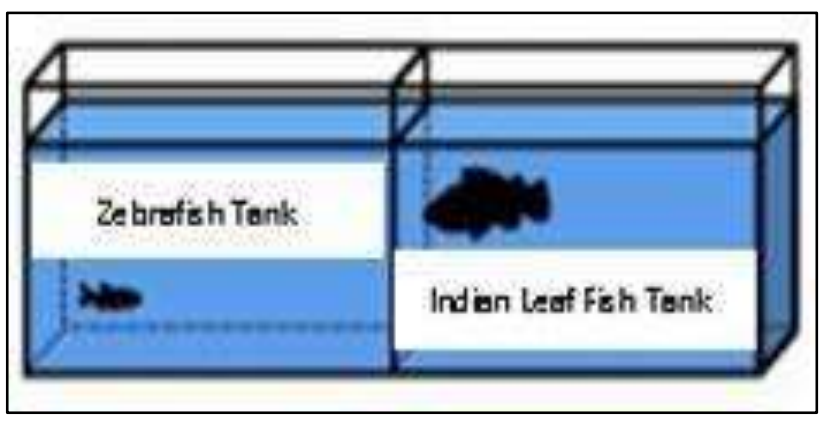

Figure 5: Predator avoidance test

Piato AL et al determined the effects of UCS (Unpredictable Chronic Stress) protocol during 7 or 14 days on behavioural and physiological parameters. The effects of stress were assessed in relation to anxiety and exploratory behaviour, memory, expression of corticotrophin-releasing factor (CRF) and glucocorticoid receptor (GR), and cortisol levels. UCS protocol enhanced the anxiety levels, diminished cognitive function, and raised CRF whereas decreased GR expression. Furthermore, zebrafish subjected to 7 or 14 days of UCS protocol presented increased cortisol levels. This is a complementary model for learning the neurobiology and the effects of chronic stress in behavioural and physiological parameters. Additionally, this protocol is less time consuming than regular rodent models usually used to study chronic stress. These outcomes endorse UCS in zebrafish as an adequate model to preclinical studies of stress, although further studies are warranted to determine its predictive validity. ${ }^{11}$

Anxiolytic effects of nicotine have been accepted in studies with rodents and humans. Understanding the neural basis of nicotine-induced anxiolysis can benefit both in developing better aids for smoking cessation besides the potential development of novel nicotinic ligands for treating anxiety. Levin ED et al assessed whether a zebrafish model of anxiety would be sensitive to nicotine. When zebrafish are positioned in a novel environment, they dive to the bottom of the tank. In the wild, diving could benefit to escape predation. The investigators verified the anxiolytic effect of nicotine on the novelty-elicited diving response and subsequent habituation. Zebrafish placed in a novel tank spent the bulk of time at the bottom third of the tank during the first minute of a 5-min session and later show a gradual diminution in time spent at the tank bottom. Nicotine treatment at $100 \mathrm{mg} / \mathrm{l}$ for 3 minutes by immersion before testing produced a noteworthy reduction in diving throughout the session, while $50 \mathrm{mg} / \mathrm{l}$ was effective during the first minute when the greatest bottom dwelling was seen in controls. Nicotine effects were reversed by the nicotinic antagonist mecamylamine given along with nicotine, but not when administered shortly before the test session after prior nicotine dosing. This suggests that the effect of nicotine on diving was because of the net stimulation at nicotinic receptors, an effect that is blocked by mecamylamine; and that once invoked, this effect is no longer dependent on continuing activation of nicotinic receptors. Nicotine-induced anxiolytic effects can be modeled in the zebrafish. This preparation will help in the investigation of the molecular bases of this effect. ${ }^{12}$

Collectively, results confirm zebrafish as a valid, reliable, and model of stress and affective disorders.

\section{Depression and antidepressants}

Supplementing genetic and experimental manipulations, pharmacological models are also extensively used in brain research. For example, depression-like behaviours in humans and rodents can be induced by reserpine, which diminishes brain monoamines by irrevocably blocking the vesicular monoamine transporter. The drug evokes strong pro-depressant effects in humans, besides causing hypo activity, motor stereotypies, lethargy, and anhedonia in rodent. ${ }^{13}$ Reserpine treatment and related neurochemical and behavioural deficits are frequently used as a model of depression in rodents, but can likewise induce depressionlike behaviour in zebrafish (including hypolocomotion and disrupted shoaling, resembling motor retardation and social withdrawal symptoms observed in clinical depression).

However, d-amphetamine enhances brain monoamines' levels, and leads to hyperactivity and anxiety. Though, the effects of these agents on behaviour and in relation to monoamine levels continue to be poorly understood, compelling further experimental studies to comprehend their psychotropic action. Kyzar E et al studied the acute and long-term effects of reserpine and d-amphetamine on zebrafish behaviour in the novel tank test. Largely, damphetamine (5 and $10 \mathrm{mg} / \mathrm{L}$ ) evokes anxiogenic-like effects in zebrafish acutely, but not 7 days later. In distinction, reserpine (20 and $40 \mathrm{mg} / \mathrm{L}$ ) did not arouse obvious acute behavioural effects, but evidently reduced activity 7 days later, approximating motor retardation witnessed in depression and/or parkinson's disease. Results validate that zebrafish are exceedingly sensitive to drugs bi-directionally modifying brain monoamines, usually paralleling rodent and clinical findings. Together, this highlights the prospect of zebrafish tests to model complex brain disorders associated with monoamine dysregulation. ${ }^{13}$ 
Interestingly, antidepressants (such as selective serotonin reuptake inhibitors, SSRIs) regulate some of the mutant phenotypes, paralleling recognized effects of these drugs in altering glucocorticoid signalling and relieving stress disorders in human patients, which also endorses the translational significance of serotonergic modulation of zebrafish stress responses. $^{14}$

Sub-chronic exposure to dosages of $100 \mu \mathrm{g} / \mathrm{L}$ fluoxetine and $20 \mathrm{mg} / \mathrm{L}$ of ketamine reduced anxiety/depression-like behaviours, leading to up regulation of serotonin synthesis and elevated whole-body cortisol levels. These results demonstrate the possible efficacy of fluoxetine and ketamine coadministration. ${ }^{15}$

\section{Psychoses and antipsychotics}

Modelling neurological diseases have demonstrated to be extraordinarily difficult because of the phenotypic complexity of each disorder. The zebrafish has become a convenient model system to study abnormal neurological and behavioural activity and holds promise as a model of human disease. The growing use of adult zebrafish in behavioural studies has generated the need for new and improved protocols.

Blazina AR et al pursued to evaluate the swimming behaviour of zebrafish against a water current using the newly developed spinning task. Zebrafish were separately located in a beaker comprising a spinning magnetic stirrer and their latency to be swept into the whirlpool was documented. The investigators categorized that larger fish $(>4 \mathrm{~cm})$ and lower r.p.m. reduced the swimming time in the spinning task. There was also a dose-related decrease in swimming after acute treatment with haloperidol, valproic acid, clonazepam, and ethanol, which modify coordination. Essentially, at doses that reduced swimming time in the spinning task, these drugs influenced absolute turn angle (ethanol increased and the other drugs decreased), but had no effect of distance travelled in a regular water tank. These results recommend that the spinning task is a useful protocol to complement evidence to the assessment of zebrafish motor behaviour. ${ }^{16}$

While most of the disease modelling using zebrafish has made use of adults, larvae too have remarkable potential for the high-throughput screening of prospective therapeutics. The additional development of larval disease models will reinforce their ability to add to the drug screening process. Ellis LD et al have utilized zebrafish larvae to model the symptoms of bipolar disorder by treating larvae with sub-convulsive concentrations of the GABA antagonist pentylenetetrazole (PTZ). A variety of therapeutics that act on diverse targets, besides those that have been used to treat bipolar disorder, were tried against this model to evaluate its predictive value. Carbamazepine, valproic acid, and baclofen, were found to oppose several aspects of the PTZ-induced variations in activity. Lidocaine and haloperidol aggravated the PTZinduced activity changes and sulpiride had no effect. By equating the degree of phenotypic rescue with the mechanism of action of each therapeutic the investigators have presented that the low-concentration PTZ model can produce a number of intermediate phenotypes that model symptoms of bipolar disorder, may be worthwhile in modelling other disease states, and will help predict the efficacy of novel therapeutics. ${ }^{17}$

Since psychotropic drugs affect behaviour, alterations in behaviour can be used to discover psychotropic drugs. The original exemplars of most neuroactive medicines were revealed in humans, rodents and other model organisms. Most of these findings were made by chance, but the development of behaviour based drug discovery can be made more methodical and efficient. Fully automated platforms for investigating the behaviour of embryonic zebrafish capture digital video recordings of animals in each individual well of a 96-well plate before, during, and after a series of stimuli. By merging the in vivo relevance of behaviour-based phenotyping with the scale and automation of modern drug screening technologies, systematic behavioural barcoding characterizes a means of discovering psychotropic drugs and provides an authoritative, organized methodology for unscrambling the complexities of vertebrate behaviour. ${ }^{18}$

Schizophrenia is a severe mental illness characterized by positive and negative symptoms and cognitive deficits. Decrease in glutamatergic neurotransmission by NMDA receptor antagonists simulates symptoms of schizophrenia. Modeling social interaction and cognitive impairment in animals can be of immense help in the effort to develop innovative treatments for negative and cognitive symptoms of schizophrenia. Studies have established that these behavioural variations are, in some cases, sensitive to remediation by antipsychotic drugs. The zebrafish has been suggested as a candidate to evaluate the in vivo effects of numerous drugs and to discover new pharmacological targets. Seibt KJ et al investigated the capability of antipsychotic drugs to reverse schizophrenia-like symptoms induced by the NMDA receptor antagonist MK-801(dizocilpine). Results exhibited that MK-801 (5 $\mu \mathrm{M})$ given pre-training delayed memory formation while both atypical antipsychotics sulpiride $(250 \mu \mathrm{M})$ and olanzapine $(50 \mu \mathrm{M})$ improved MK-801-induced amnesia. An identical change was detected in the social interaction task, where atypical antipsychotics reversed the MK-801-induced social interaction deficit; however, the typical antipsychotic haloperidol $(9 \mu \mathrm{M})$ was ineffective to reverse those behavioural deficits. Hence, MK-801-treated zebrafish showed some behavioural features witnessed in schizophrenia, such as cognitive and social interaction deficits, which were reverted by current available atypical drugs. ${ }^{19}$

Additionally, these same investigators categorized the behavioural effects of MK-801 and investigated the effect of typical and atypical antipsychotic treatments on locomotors activity as well on the hyper locomotion 
induced by MK-801 in zebrafish. MK-801 (20 microM) increased the locomotors behaviour as measured by the amount of line crossings, distance travelled, and the mean speed in the tank test after 15, 30, and 60 minutes of exposure. All tested antipsychotics counteracted MK-801induced hyperactivity on all parameters analysed and at doses that, given alone, had no effect on spontaneous locomotors activity. The results recommend a similar profile between typical and atypical antipsychotics in the reversal of locomotors disorders induced by MK-801. Likewise, an anxiolytic effect was verified at 30 and 60 min of MK-801 exposure that was not reversed by antipsychotics tested in this work. Furthermore, olanzapine, which alone caused an anxiolytic response, when given with MK-801 potentiated the latter's effect on anxiety. Accordingly it validated the value of the zebrafish, a simple to use animal model, in evolving some behavioural features observed in schizophrenia, which may indicate a new approach for drug screening. ${ }^{20}$

\section{Mood stabilisers/lithium}

After more than 60 years from its therapeutic discovery, lithium still remains the exemplary treatment for bipolar disorder and has been extensively utilized as a mood stabilizer because of its capability to diminish manic and depressive episodes, efficacy in long-term mood stabilization, and usefulness in decreasing patient suicide risks. There has been a great effort to describe lithium cellular and system actions, directing to improve treatment effectiveness and diminish side effects. In order to entirely evaluate the therapeutic and toxicological effects of lithium, diverse experimental models have been proposed.

In spite of the growing interest about the effects of antidepressants and anxiolytics on behavioural responses in zebrafish, insufficient studies have been implemented assessing the actions of lithium on this experimental model. Studies have shown that drugs used for treatment of mood disorders, such as lithium, are able to modulate circadian rhythm. Lithium chloride $(\mathrm{LiCl})$ promoted a 0.7 $\mathrm{h}$ lengthening of the circadian period in a specific zebrafish line, able to detect maturing and developmental activity of the circadian clock. $\mathrm{LiCl}$ and $\mathrm{LiCl}$ plus forskolin treatments promoted a significant increase in the pigmentation in zebrafish embryos, due to synthesis of melanin in neural crest-derived melanophores.

So far only an inadequate number of studies evaluated the toxicological impact of lithium ion zebrafish development and demonstrated morphological, physiological, and behavioural effects that may be informative regarding human findings. Additional studies devoted to characterize and assess the underlying mechanisms of the toxic effects and the potential impact of exposure on developing and adult individuals are necessary to establish safe clinical management guidelines for women with bipolar disorder of childbearing age and safety disposal guidelines for pharmaceutical neuroactive compounds. $^{21}$

\section{Alcohol research}

Zebrafish are at the forefront of neurobiological research and have been gaining acceptance as a feasible and effective behavioural model in a range of research applications. This model turn out to be even more attractive bearing in mind the behavioural changes that follow exposure to compounds that are water-soluble.

Alcohol abuse and dependence are a rapidly growing problem with scarce treatment options available. The zebrafish has become a popular animal model for behavioural neuroscience. This species may be appropriate for investigating the effects of alcohol on the vertebrate brain.

As such, numerous studies have associated both acute and chronic ethanol exposure in the modulation of zebrafish behaviour. Within this field there seems to be a common drift across multiple studies. As with many drugs ethanol appears to influence behaviour in a dose-dependent manner. ${ }^{22}$

Alcohol tolerance is often observed following chronic exposure to low concentrations of alcohol. Alcohol sensitization also called reverse tolerance (a progressive increase in the effect of alcohol over time) is frequently detected ensuing recurrent separate exposures to higher concentrations of alcohol. These two phenomena might underlie the development and maintenance of alcohol addiction. The phenotypical characterization of these responses in zebrafish may be the first important steps in establishing this species as a tool for the analysis of the molecular and neurobiological mechanisms underlying human alcohol addiction. ${ }^{23}$

Considering these efforts, the activity of adult zebrafish is normally computed using indirect activity measures that are either scored manually or identified automatically from the fish trajectory. The exploration of such activity measures has produced vital understanding into the effect of acute ethanol exposure on individual and social behaviour of this vertebrate species. Bartolini et al have recently established a tracking algorithm that reconstructs fish body shape to explore the effect of acute ethanol administration on zebrafish tail-beat motion in terms of amplitude and frequency. Their research demonstrate a significant effect of ethanol on the tail-beat amplitude as well as the tail-beat frequency, both of which were found to strongly decrease for high ethanol concentrations. Such a direct measurement of zebrafish motor functions is in agreement with evidence based on indirect activity measures, proposing a corresponding perspective in behavioural screening. ${ }^{24}$

Luchiari et al analysed the effects of alcohol on performance of zebrafish in a recently developed latent 
learning paradigm. It was found that acute exposure to $1.00 \%$ alcohols after chronic freshwater disrupted learning performance, so did exposure to freshwater after chronic alcohol treatment (withdrawal). It was also found that exposure to chronic alcohol diminished the effect of subsequent acute alcohol suggesting development of tolerance. These results demonstrate that analysis of learning performance of zebrafish allows detection of alcohol-induced functional changes. The simplicity and scalability of the employed task also imply the utility of the zebrafish in high throughput drug screens. ${ }^{25}$

Even Mathur et al measured the effects of both acute and chronic ethanol exposure on anxiety-like behaviours in zebrafish, using two behavioural paradigms, the novel tank diving test and the light/dark choice assay. Acute ethanol exposure exerted significant dose-dependent anxiolytic effects. Nevertheless, withdrawal from repetitive intermittent ethanol exposure disabled recovery from heightened anxiety. These results validate that zebrafish display diverse anxiety-like behavioural responses to acute and chronic ethanol exposure, which are remarkably similar to these effects of alcohol in humans. Due to the accessibility of zebrafish to high throughput screening, results advocate that genes and small molecules identified in zebrafish will be of relevance to understand how acute versus chronic alcohol exposure have opposing effects on the state of anxiety in humans. $^{26}$

\section{Nootropics/cognitive enhancers}

Piracetam, a derivative of $\gamma$-aminobutyric acid, exerts memory-enhancing and mild anxiolytic effects in human and rodent studies. To scrutinize the drug's behavioural profile further, Grossman et al measured its effects on behavioural and endocrine (cortisol) responses of adult zebrafish. Generally, acute piracetam did not affect zebrafish novel tank and light-dark box behaviour at mild doses $(25-400 \mathrm{mg} / \mathrm{L})$, but produced nonspecific behavioural inhibition at $700 \mathrm{mg} / \mathrm{L}$. No effects on cortisol levels or inter-/intra-session habituation in the novel tank test were observed for acute or chronic mild non-sedative dose of $200 \mathrm{mg} / \mathrm{L}$. however, fish exposed to chronic piracetam at this dose performed significantly better in the cued learning plus-maze test. This observation parallels clinical and rodent literature on the behavioural profile of piracetam, supporting the utility of zebrafish paradigms for testing nootropic agents. ${ }^{27}$

\section{Epilepsy and antiepileptics}

Rodent seizure models have considerably added to our basic understanding of epilepsy. Yet, medically intractable forms of epilepsy continue and the essential mechanisms underlying this disease remain unclear. Baraban SC et al have established that seizures can be produced in a simple vertebrate system e.g. zebrafish larvae. Exposure to a common convulsant agent (pentylenetetrazole, PTZ) induced a stereotyped and concentration-dependent sequence of behavioural changes ending in clonus-like convulsions. Extracellular recordings from fish optic tectum revealed ictal and interictal-like electrographic discharges subsequent to the application of PTZ, which could be blocked by tetrodotoxin or glutamate receptor antagonists. Epileptiform discharges were inhibited by generally used antiepileptic drugs, valproate and diazepam, in a concentration-dependent manner. Up-regulation of c-fos expression was also observed in CNS structures of zebrafish exposed to PTZ. Taken together, these results exhibit that chemically-induced seizures in zebrafish demonstrate behavioural, electrographic, and molecular changes that would be expected from a rodent seizure model. Consequently, zebrafish larvae signify a powerful new system to study the underlying basis of seizure generation, epilepsy and epileptogenesis. ${ }^{28}$

Mussulini BH et al performed a detailed temporal behaviour profile characterization of PTZ-induced seizure in adult zebrafish. The behavioural profile during $20 \mathrm{~min}$ of PTZ immersion (5, 7.5, 10, and $15 \mathrm{mM}$ ) was characterized by stages defined as scores: (0) short swim, (1) increased swimming activity and high frequency of opercular movement, (2) erratic movements, (3) circular movements, (4) clonic seizure-like behaviour, (5) fall to the bottom of the tank and tonic seizure-like behaviour, (6) death. Animals exposed to distinct PTZ concentrations presented different seizure profiles, intensities and latencies to reach all scores. Pre-treatment with diazepam significantly attenuated seizure severity. Finally, the brain PTZ levels in adult zebrafish immersed into the chemoconvulsant solution at 5 and $10 \mathrm{mM}$ were comparable to those described for the rodent model, with a peak after a 20-min of exposure. The PTZ brain levels observed after 2.5-min PTZ exposure and after 60-min removal from exposure were similar. Altogether, these results showed a detailed temporal behavioural characterization of a PTZ epileptic seizure model in adult zebrafish. These behavioural analyses and the simple method for PTZ quantification could be considered as important tools for future investigations and translational research. $^{29}$

Additionally, Gupta $\mathrm{P}$ et al investigated the effect of established anticonvulsants, such as valproic acid, carbamazepine, gabapentin, diazepam, lacosamide and pregabalin against pentylenetetrazole $(6 \mathrm{mM})$ seizures in adult zebrafish. Different phases of seizures (increase swim activity, rapid whirlpool-like circling swim behaviour and brief clonus-like seizures leading to loss of posture) were elicited in zebrafish on exposure for 15 minutes to $6 \mathrm{mM}$ pentylenetetrazole. The exposure of zebrafish to an increasing concentration of the anticonvulsants alongside $6 \mathrm{mM}$ pentylenetetrazole showed concentration-dependent elevation of seizure latency against pentylenetetrazole-induced seizures except for pregabalin, which failed to produce any anticonvulsant activity in zebrafish. Additionally the proconvulsant activity of caffeine was also estimated using suboptimal 
concentration (4 $\mathrm{mM}$ ) of pentylenetetrazole in adult zebrafish. Reduction in seizure latency of different phases of seizures was detected with increasing concentration of caffeine compared with its respective control group. In view of the above findings, the results of the study suggested that adult zebrafish produce the expected anticonvulsive and proconvulsive effects and could potentially be used as a screen in future epilepsy research. $^{30}$

\section{Autism spectrum disorder (ASD)}

Autism spectrum disorder characterizes additional collection of serious behavioural deficits, affecting $\sim 1$ $2 \%$ of the general population. While the prevalence of ASD is lower than depression or anxiety (which affect $>10-15 \%$ of the adult population worldwide), autism causes a huge amount of human suffering, which (if expressed in patient-years; i.e., the number of patients multiplied by the length of time for which the patient suffers from the disease) signifies a pressing unmet medical need. In addition to severe behavioural and cognitive impairments, ASD is characterized by high $(\sim 90 \%)$ heritability, representing one of the most heritable brain disorders in humans. ${ }^{31}$

Animal (rodent) models of ASD-like behaviour are widely used to study genetics, circuitry and molecular mechanisms of ASD. The evolutionarily preserved nature of social behaviour and its molecular pathways proposes that alternative experimental models can be established to supplement and boost the existing rodent ASD paradigms. The zebrafish is rapidly becoming a popular model organism in neuroscience and biological psychiatry to study brain function, model human brain disorders and explore their genetic or pharmacological modulation. Representing highly social animals, zebrafish emerge as a strong potential model organism to study normal and pathological social phenotypes, as well as several other ASD-like symptoms. ${ }^{32}$

Thus, as a result of over four decades of developmental biology research with zebrafish, this species has become one of the most powerful vertebrate tools, and may therefore offer uniquely efficient answers to the conundrum of the developmental abnormalities associated with ASD. ${ }^{34}$

\section{Drug abuse and withdrawal}

Drug-induced disorders are a significant area of biomedical research. One of the main examples is addiction, a prevalent disorder frequently associated with drug abuse. Supplementing traditional (rodent) models, zebrafish are effective translational models to study reward and drug abuse. Both larval and adult zebrafish show high sensitivity to various drugs of abuse, as well as tolerance, clear preference (reward stimuli) for these agents, and withdrawal symptoms. ${ }^{33,34}$
Rising evidence involves the zebrafish as a promising model species for reward and addiction research. Modeling drug abuse-related behaviour in both adult and larval zebrafish produced a wealth of clinically translatable data, also demonstrating their sensitivity to numerous drugs of abuse and the ability to develop tolerance. Quite a few studies have also applied withdrawal paradigms to model the adverse effects of drug abuse in zebrafish. ${ }^{35}$

For instance, the effects of alcohol in zebrafish have been studied for more than a dozen years, revealing numerous behavioural changes in fish that resemble those seen in rodents and humans. ${ }^{36,41-43}$ Acute alcohol reduces zebrafish fear/anxiety at lower doses whereas higher doses induce lethargy and sedation. Additionally, chronic alcohol exposure also demonstrates parallels between zebrafish and mammals. Similarly, withdrawal from chronic alcohol exposure leads to several behavioural and physiological abnormalities that resemble anxiogenic withdrawal symptoms in humans or rodents.

Though considered as 'dangerous' drugs of abuse, hallucinogenic agents frequently have low-to-mild addictive properties, and are of importance because of their strong psychotropic effects and potential for treating brain disorders, including depression, anxiety, and posttraumatic stress. ${ }^{37,38}$

Strongly affecting human and animal CNS, several hallucinogenic drugs (such as serotonergic psychedelic, glutamatergic dissociative and cholinergic deliriant agents) have lately been screened in zebrafish. ${ }^{39}$ These studies not only showed noticeable behavioural and physiological responses to these psychoactive drugs (similar to those in humans) but also established remarkable parallels amongst the pharmacological profiles of drugs in zebrafish, humans, and rodents.

Cachat $\mathbf{J}$ et al have studied the effects of ethanol, diazepam, and morphine and caffeine withdrawal on zebrafish behaviour. Generally, discontinuation of ethanol, diazepam and morphine produced anxiogeniclike behavioural or endocrine responses, demonstrating the utility of zebrafish in translational research of withdrawal syndrome. ${ }^{34}$

Ketamine is a non-competitive glutamatergic antagonist used to induce sedation and analgesia. In sub-anaesthetic doses, it induces hyperlocomotion, impairs memory and evokes stereotypic circling in rodents. Zebrafish has emerged as a promising new animal model to screen the effects of psychotropic compounds. Reihl $\mathrm{R}$ et al have investigated the effects of sub-anesthetic doses of ketamine on anxiety, locomotion, habituation and social behaviour of adult zebrafish. Acute 20 minutes exposure to 20 and $40 \mathrm{mg} / \mathrm{L}$ of ketamine reduced anxiety, impaired intra-session habituation, evoked circular swimming and disrupted zebrafish shoaling. Additionally, ketamine reduced whole-body cortisol levels and elevated brain c- 
fos expression in zebrafish. These findings demonstrate the sensitivity of zebrafish to behavioural and physiological effects of sub-anesthetic doses of ketamine, further supporting the utility of this species as a model for neuropharmacological research, including testing ketamine and related drugs. ${ }^{40}$

An indole alkaloid, ibogaine is the principal psychoactive component of the iboga plant, used by indigenous peoples in West Africa for centuries. Modulating multiple neurotransmitter systems, the drug is a potent hallucinogen in humans, although its psychotropic effects remain poorly understood. Cachat $\mathrm{J}$ et al exposed adult zebrafish to 10 and $20 \mathrm{mg} / \mathrm{L}$ of ibogaine, testing them in the novel tank, light-dark box, open field, mirror stimulation, social preference and shoaling tests. In the novel tank test, the zebrafish natural diving response (geotaxis) was reversed by ibogaine, inducing initial top swimming followed by bottom dwelling. Ibogaine also attenuated the innate preference for dark environments (scototaxis) in the light-dark box test. These results support the high sensitivity in zebrafish models, dosedependently affecting multiple behavioural domains and strongly support the developing utility of aquatic models in hallucinogenic drug research. ${ }^{41}$

Mescaline and phencyclidine (PCP) are potent hallucinogenic agents affecting human and animal behaviour. Kyzar EJ et al scrutinized the effects of mescaline and PCP in numerous zebrafish paradigms, comprising the novel tank, open field and shoaling tests. Mescaline and PCP dose-dependently augmented top activity in the novel tank test, also decreasing immobility and disturbing the patterning of zebrafish swimming. Largely, these studies indicate high sensitivity of zebrafish models to hallucinogenic compounds with complex behavioural and physiological effects. ${ }^{42}$

Lysergic acid diethylamide (LSD) is a powerful hallucinogenic drug that intensely affects animal and human behaviour. Quite a few behavioural paradigms (the novel tank, observation cylinder, light-dark box, open field, T-maze, social preference and shoaling tests), as well as modern video-tracking tools and whole-body cortisol assay were utilized to describe the effects of acute LSD in zebrafish. On the whole, findings in these studies show sensitivity of zebrafish to LSD action, and support the use of zebrafish models to study hallucinogenic drugs of abuse. ${ }^{43}$

Serotonin syndrome (SS) is a severe fatal disorder linked with raised brain serotonergic function. With the growing use of serotonergic drugs, SS distresses a large portion of general population, becoming a foremost biomedical concern. SS-like behaviours have also been described in animals subsequent to the administration of serotonergic drugs. While clinical and rodent studies have provided substantial understanding into the etiology of SS, its precise mechanisms and risk factors remain poorly understood. The necessity to develop more effective psychotropic drugs also entails extensive high-throughput screening of novel compounds using sensitive in-vivo tests. The use of zebrafish in neuroscience research is speedily escalating due to their homology to humans, robust behavioural and physiological responses, genetic tractability, and low costs. Overall, zebrafish exposed to serotonergic agents and their combinations exhibit a characteristic top dwelling (surfacing behaviour) and hypolocomotion which may represent potential markers of SS-like states in zebrafish. This behaviour in zebrafish models positively correlates with brain concentrations of serotonin, suggesting the developing utility of zebrafish for studying SS. Forthcoming research is anticipated to foster high-throughput screening of drug interactions, and pharmacogenetics studies identifying zebrafish mutations implicated in pathological SS-like states. ${ }^{44}$

\section{Pain/analgesics}

Acute and chronic pain conditions are frequently incapacitating, causing severe physiological, emotional distress and economic burden and disturb a great percentage of the global population. Nevertheless, the development of therapeutic analgesic agents based primarily on targeted drug development has been essentially unsuccessful. An alternative methodology to analgesic development would be to develop low cost, high throughput, untargeted animal based behavioural screens that model multifaceted nociceptive behaviours to screen for analgesic compounds. Curtright A et al have described the development of a behavioral based assay in zebrafish larvae that is effective in identifying small molecule compounds with analgesic properties. Modeling thermal hyperalgesia, the addition of the noxious inflammatory compound and TRPA1 agonist allyl isothiocyanate sensitized heat aversion and reversed cool aversion leading larvae to avoid rearing temperature in favour of otherwise acutely aversive cooler temperatures. The investigators have demonstrated that small molecules with known analgesic properties are able to inhibit acute and/or sensitized temperature aversion. ${ }^{45}$

Nociception is the sensory mechanism used to perceive indications that can harm an organism. The understanding of the neural networks and molecular controls of the reception of pain remains a continuing challenge. Mice and rat animal models have been comprehensively used for nociception studies. However, the study of pain and noiception in these organisms can be rather laborious, costly and time consuming. Malafloglia $\mathrm{V}$ et al have charted the explanations why zebrafish presents a novel and attractive model for studying pain reception and responses and the most interesting findings in the study of nociception that have been obtained using the zebrafish model. $^{46}$

Gonzalez-Nunez V et al have described the zebrafish opioid system, a unique experimental method to unravel the molecular mechanisms that underlie opioid activity. They have established zebrafish opioid receptors and 
peptides. Their expression patterns during development and in the adult organism have been identified. Additionally, their pharmacological profiles and biochemical properties have been determined. Moreover, developmental studies in the zebrafish have generated valuable information about the developmental roles of the opioid receptors. Building on these findings, it has been demonstrated that the zebrafish opioid receptors and peptides present molecular, pharmacological, and biochemical profiles that are primarily comparable to those of their mammalian counterparts and from which results can hence be extrapolated to higher vertebrates. Accordingly the zebrafish represents a straightforward model to study opioid activity, and can be very beneficial not only for the investigation of the complex endogenous systems that regulate the action of opioid agents but also for in vivo tests of novel analgesic drugs. ${ }^{47}$

\section{CONCLUSION}

Though, similar to any model organism, the zebrafish also has its limitations. For example, a documented limitation in zebrafish genetics is the dearth of well-characterized inbred strains. Hence, forthcoming efforts may be desirable to increase the number of existing zebrafish strains and advance our understanding of the strain variances in fish neural phenotypes.

In general, no experimental animal model can target the entireness of any complex brain disorder detected clinically in human patients. Several questions remain to be addressed in future zebrafish studies.

Several significant neuropsychiatric domains were not discussed in-depth here because of their coverage by current comprehensive literature elsewhere. Similarly, aging-related psychiatric disorders and cognitive decline are getting increasing acknowledgment in clinical psychiatry, and have recently been successfully modelled in zebrafish.

In summary, zebrafish models are becoming a significant tool anticipated to progress neuroscience and neurogenetics. Zebrafish sensitivity to all major neurotropic drugs and the capability to respond to them in an analogous manner as humans, support their usefulness for pharmacological research.

Thus, zebrafish prove to be progressively worthwhile in translational brain research, and are well matched to meet the fast developing challenges of this field.

Funding: No funding sources Conflict of interest: None declared

Ethical approval: Not Required

\section{REFERENCES}

1. Norton WH. Toward developmental models of psychiatric disorders in zebrafish. Front Neural Circuits. 2013;7:79.

2. Maximino C, Silva RX, da Silva Sde N, Rodrigues Ldo S, Barbosa H, de Carvalho TS, et al. Nonmammalian models in behavioral neuroscience: consequences for biological psychiatry. Front Behav Neurosci. 2015;9:233.

3. Kalueff AV, Stewart AM, Gerlai R. Zebrafish. As an emerging model for studying complex brain disorders. Trends Pharmacol Sci. 2014;35:63-75.

4. Panula P, Chen YC, Priyadarshini M, Kudo H, Semenova S, Sundvik M, et al. The comparative neuroanatomy and neurochemistry of zebrafish CNS systems of relevance to human neuropsychiatric diseases. Neurobiol Dis. 2010;40:46-57.

5. Ali S, Champagne DL, Spaink HP, Richardson MK. Zebrafish embryos and larvae: a new generation of disease models and drug screens. Birth Defects Res C Embryo Today. 2011;93:115-33.

6. Stewart A, Wu N, Cachat J, Hart P, Gaikwad S, Wong K, et al. Pharmacological modulation of anxiety-like phenotypes in adult zebrafish behavioral models. Prog Neuropsychopharmacol Biol Psychiatry. 2011;35:1421-31.

7. Cachat J, Stewart A, Grossman L, Gaikwad S, Kadri $\mathrm{F}$, Chung KM, et al. Measuring behavioral and endocrine responses to novelty stress in adult zebrafish. Nat Protoc. 2010;5:1786-99.

8. Bencan Z, Sledge D, Levin ED. Buspirone, chlordiazepoxide and diazepam effects in a zebrafish model of anxiety. Pharmacol Biochem Behav. 2009;94:75-80.

9. Egan RJ, Bergner CL, Hart PC, Cachat JM, Canavello PR, Elegante MF, et al. Understanding behavioral and physiological phenotypes of stress and anxiety in zebrafish. Behav Brain Res. 2009;205:38-44.

10. Chakravarty S, Reddy BR, Sudhakar SR, Saxena S, Das T, Meghah V, et al. Chronic unpredictable stress (CUS)-induced anxiety and related mood disorders in a zebrafish model: altered brain proteome profile implicates mitochondrial dysfunction. PLoS One. 2013;8:e63302.

11. Piato ÂL, Capiotti KM, Tamborski AR, Oses JP, Barcellos LJ, Bogo MR, et al. Unpredictable chronic stress model in zebrafish (danio rerio): behavioral and physiological responses. Prog Neuropsychopharmacol Biol Psychiatry. 2011;35:561-7.

12. Levin ED, Bencan Z, Cerutti DT. Anxiolytic effects of nicotine in zebrafish. Physiol Behav. 2007;90:54-8.

13. Kyzar E, Stewart AM, Landsman S, Collins C, Gebhardt M, Robinson K, et al. Behavioral effects of bidirectional modulators of brain monoamines reserpine and d-amphetamine in zebrafish. Brain Res. 2001;1527:108-16. 
14. Ziv L, Muto A, Schoonheim PJ, Meijsing SH, Strasser D, Ingraham HA, et al. An affective disorder in zebrafish with mutation of the glucocorticoid receptor. Mol Psychiatry. 2013;18:681-91.

15. Pittman J, Hylton A. Behavioral, endocrine, and neuronal alterations in zebrafish (danio rerio) following sub-chronic coadministration of fluoxetine and ketamine. Pharmacol Biochem Behav. 2015;S0091-3057:30051-4.

16. Blazina AR, Vianna MR, Lara DR. The spinning task: a new protocol to easily assess motor coordination and resistance in zebrafish. Zebrafish. 2013; 10:480-5.

17. Ellis LD, Soanes KH. A larval zebrafish model of bipolar disorder as a screening platform for neurotherapeutics. Behav Brain Res. 2012;233:450-7.

18. Kokel D, Peterson RT. Using the zebrafish photomotor response for psychotropic drug screening. Methods Cell Biol. 2011;105:517-24.

19. Seibt KJ, Piato AL, da Luz Oliveira R, Capiotti KM, Vianna MR, Bonan CD. Antipsychotic drugs reverse MK-801-induced cognitive and social interaction deficits in zebrafish (danio rerio). Behav Brain Res. 2011;224:135-9.

20. Seibt KJ, Oliveira Rda L, Zimmermann FF, Capiotti KM, Bogo MR, Ghisleni G, et al. Antipsychotic drugs prevent the motor hyperactivity induced by psychotomimetic MK-801 in zebrafish (danio rerio). Behav Brain Res. 2010;214:417-22.

21. Siebel AM, Vianna MR, Bonan CD. Pharmacological and toxicological effects of lithium in zebrafish. ACS Chem Neurosci. 2014;5:468-76.

22. Echevarria DJ, Toms CN, Jouandot DJ. Alcoholinduced behavior changes in zebrafish models. Rev Neurosci. 2011;22:85-93.

23. Tran S, Gerlai R. Recent advances with a novel model organism: alcohol tolerance and sensitization in zebrafish (Danio rerio). Prog Neuropsychopharmacol Biol Psychiatry. 2014;55:87-93.

24. Bartolini T, Mwaffo V, Butail S, Porfiri M. Effect of acute ethanol administration on zebrafish tail-beat motion. Alcohol. 2015;49:721-5.

25. Luchiari AC, Salajan DC, Gerlai R. Acute and chronic alcohol administration: effects on performance of zebrafish in a latent learning task. Behav Brain Res. 2015;282:76-83.

26. Mathur P, Guo S. Differences of acute versus chronic ethanol exposure on anxiety-like behavioral responses in zebrafish. Behav Brain Res. 2011;219:234-9.

27. Grossman L, Stewart A, Gaikwad S, Utterback E, Wu N, Dileo J, et al. Effects of piracetam on behavior and memory in adult zebrafish. Brain Res Bull. 2011;85:58-63.

28. Baraban SC, Taylor MR, Castro PA, Baier $\mathrm{H}$. Pentylenetetrazole induced changes in zebrafish behavior, neural activity and c-fos expression. Neuroscience. 2005;131:759-68.
29. Mussulini BH, Leite CE, Zenki KC, Moro L, Baggio $\mathrm{S}$, Rico EP, et al. Seizures induced by pentylenetetrazole in the adult zebrafish: a detailed behavioral characterization. PLoS One. 2013;8:e54515.

30. Gupta P, Khobragade SB, Shingatgeri VM. Effect of various antiepileptic drugs in zebrafish ptz-seizure model. Indian J Pharm Sci. 2014;76:157-63.

31. Zafeiriou DI, Ververi A, Dafoulis V, Kalyva E, Vargiami E. Autism spectrum disorders: the quest for genetic syndromes. Am J Med Genet B Neuropsychiatr. Genet. 2013;162B:327-66.

32. Stewart AM, Nguyen M, Wong K, Poudel MK, Kalueff AV. Developing zebrafish models of autism spectrum disorder (ASD). Prog Neuropsychopharmacol Biol Psychiatry. 2014;50:27-36.

33. Stewart A, Wong K, Cachat J, Gaikwad S, Kyzar E, $\mathrm{Wu} \mathrm{N}$, et al. Zebrafish models to study drug abuserelated phenotypes. Rev Neurosci. 2011;22:95-105.

34. Cachat J, Canavello P, Elegante M, Bartels B, Hart P, Bergner $\mathrm{C}$, et al. Modeling withdrawal syndrome in zebrafish. Behav Brain Res. 2010;208:371-6.

35. Collier AD, Echevarria DJ. The utility of the zebrafish model in conditioned place preference to assess the rewarding effects of drugs. Behav Pharmacol. 2013;24:375-83.

36. Tran S, Gerlai R. Time-course of behavioural changes induced by ethanol in zebrafish (danio rerio). Behav. Brain Res. 2013;252:204-13.

37. Nutt DJ, King LA, Nichols DE. Effects of Schedule I drug laws on neuroscience research and treatment innovation. Nat Rev Neurosci. 2013;14:577-85.

38. Stewart AM, Kalueff AV. Controlled substances and innovation of biomedicine. Nat Rev Neurosci. 2013; $14: 877$.

39. Neelkantan N, Mikhaylova A, Stewart AM, Arnold R, Gjeloshi V, Kondaveeti D, et al. Perspectives on zebrafish models of hallucinogenic drugs and related psychotropic compounds. ACS Chem Neurosci. 2013;4:1137-50.

40. Riehl R, Kyzar E, Allain A, Green J, Hook M, Monnig L, et al. Behavioral and physiological effects of acute ketamine exposure in adult zebrafish. Neurotoxicol Teratol. 2011;33:658-67.

41. Cachat J, Kyzar EJ, Collins C, Gaikwad S, Green J, Roth A, et al. Unique and potent effects of acute ibogaine on zebrafish: the developing utility of novel aquatic models for hallucinogenic drug research. Behav Brain Res. 2013;236:258-69.

42. Kyzar EJ, Collins C, Gaikwad S, Green J, Roth A, Monnig L, et al. Effects of hallucinogenic agents mescaline and phencyclidine on zebrafish behavior and physiology. Prog Neuropsychopharmacol Biol Psychiatry. 2012;37:194-202.

43. Grossman L, Utterback E, Stewart A, Gaikwad S, Chung KM, Suciu C, et al. Characterization of behavioral and endocrine effects of LSD on zebrafish. Behav Brain Res. 2010;214:277-84. 
44. Stewart AM, Cachat J, Gaikwad S, Robinson KS, Gebhardt M, Kalueff AV. Perspectives on experimental models of serotonin syndrome in zebrafish. Neurochem Int. 2013;62:893-902.

45. Curtright A, Rosser M, Goh S, Keown B, Wagner E, Sharifi J, et al. Modeling nociception in zebrafish: a way forward for unbiased analgesic discovery. PLoS One. 2015;10:e0116766.
46. Malafoglia V, Bryant B, Raffaeli W, Giordano A, Bellipanni G. The zebrafish as a model for nociception studies. J Cell Physiol. 2013;228:1956-66.

47. Gonzalez-Nunez V, Rodríguez RE . The zebrafish: model to study the endogenous mechanisms of pain. ILAR J. 2009;50:373-86.

Cite this article as: Bhandari PR. Zebrafish: a novel model in neuropsychopharmacological research. Int J Basic Clin Pharmacol 2016;5:546-57. 